\title{
Modal Analysis of Anapoles, Internal Fields and Fano Resonances in Dielectric Particles
}

\author{
Rémi Colom, ${ }^{1,2,{ }^{*}}$ Ross McPhedran, ${ }^{2}$ Brian Stout, ${ }^{1}$ Nicolas \\ BONOD, ${ }^{1, *}$ \\ ${ }^{1}$ Aix Marseille Univ, CNRS, Centrale Marseille, Institut Fresnel, 13013 Marseille, France \\ ${ }^{2}$ Institute for Photonics and Optical Science (IPOS), University of Sydney NSW 2006 Australia \\ *remi.colom@fresnel.fr;nicolas.bonod@fresnel.fr
}

\begin{abstract}
All-dielectric nanostructures have aroused strong interest because of their potential to trap light at subwavelength scales and to yield strong internal electric or magnetic field enhancements. Optimizing the internal fields appears as a crucial challenge for enhancing light matter interactions in all-dielectric nanostructures. Mie resonators host radiationless states called anapoles associated to a pronounced minimum of light scattering. However, the question is to know whether these radiationless states maximize the internal field intensities. Here we use a modal expansion of the internal and external fields to demonstrate that anapoles in dielectric Mie resonators result from a Fano resonance produced by the interference between two mode contributions with low and high quality factors plus an additional non-resonant term. A modal expansion of the internal field enhancement averaged over the whole scatterer volume shows that the maximum of the internal field enhancement does not occur at the anapole frequency but at the real part of the eigen-frequency associated with the high quality factor. This analysis is carried out for both electric and magnetic modes of the dielectric scatterer and evinces that a larger internal field enhancement is found for the first magnetic dipole resonance even though this resonance does not feature an anapole behavior.
\end{abstract}

\section{Introduction}

The field of resonant nanophotonics aims at enhancing light matter interactions at nanometer scales due to the resonant coupling of light with subwavelength sized particles [1-3]. The excitation of plasmonic and Mie resonances in such particles permits the enhancement of light scattering as well as the near field in the direct vicinity of the particle [4]. In the case of dielectric particles, the internal field can be strongly enhanced without loss, which opens unique opportunities in non-linear optics to generate second or third harmonics with semi-conductor particles [5-9]. It was shown in particular that the magnetic modes yield stronger confinement of the electric field intensity inside the resonant particles than the electric modes [5,10]. Recently, a very interesting phenomenon was also identified in the field of all-dielectric nanophotonics with anapoles [11-17]. Anapoles are classically defined in physics as non-radiating sources of current [18-20]. They have aroused a keen interest on account of their potential ability to enhance the light absorption in the context of dielectric resonators with promising applications in non-linear optics, light absorption and enhanced Raman scattering [21-29].

In many studies, the choice has been made to forsake the multipolar theory in spherical coordinates, a theory that is widely used for explaining Mie resonances in high refractive index materials, in favor of the multipolar theory in cartesian coordinates including the toroidal multipoles introduced by Zel'dovitch in 1958 [18-20]. In fact, the toroidal-type currents are the higher-order terms appearing in the power series expansion of the spherical electric multipoles with respect to the size of the current source. [30,31]. In this framework, anapoles occur when the electromagnetic field radiated by these toroidal multipoles cancels the field radiated by the electric cartesian multipoles. This phenomenon can also be understood by using a Quasi-Normal Mode (QNM) expansion. A QNM analysis associated with a multipolar decomposition pointed out the destructive interference between the first two modes of the electric dipolar coefficient that 
drives to a dip in the scattering spectrum [32]. The role of the first two electric modes of the electric dipolar coefficient in the excitation of anapoles in silicon scatterers was confirmed in the framework of a multipolar analysis [33]. Lastly, a toy model was developed on the analogy between optical resonators and the dynamic of a forced pendulum to study the transient internal field yielded by anapoles [15].

Here we use a modal expansion of the internal and scattered fields to demonstrate that anapoles in spherical Mie resonators arise from the fact that the scattered field features Fano-type resonances while the internal field features Lorentzian responses only. We perform the modal analysis for both electric and magnetic cases and explain why anapoles are not observed for the first resonance of the magnetic dipole. It is also pointed out that the internal electric field enhancement is larger for the first magnetic dipole resonance than for its electric dipole counterpart. The fact that the strongest field enhancement is observed in absence of anapole condition is properly predicted by our modal analysis. Results are obtained by carrying out a modal analysis of the $T$-matrix scattering operator in the multipolar Mie basis [33-35]. We believe that determining the anapole conditions in high refractive index scatterers in a widely used multipolar theory facilitates the understanding of the origin of this phenomenon.

\section{Pole expansion of the T-matrix coefficients}

\subsection{T-matrix coefficients}

Here, we are studying the anapole condition for a spherical scatterer located in vacuum with a radius $R$ and a dielectric perimittivity $\varepsilon_{s}$. Since only non-magnetic materials are considered, the refractive index possesses the following definition $n_{s}=\sqrt{\varepsilon_{s}}$. When studying light-scattering in $3 \mathrm{D}$, in particular for spherically-symmetric scatterers, it is convenient to make use of the set of Vector Partial Waves (VPWs) as a basis for the fields of the problem [36-38]. In this section, we shall consequently start by introducing the definitions used throughout this paper for the Vector Partial Waves. The outgoing VPWs are defined as follows:

$$
\begin{aligned}
& \mathbf{M}_{n, m}^{(+)}(k \mathbf{r})=h_{n}^{(+)}(k r) \mathbf{X}_{n, m}(\theta, \phi), \\
& \mathbf{N}_{n, m}^{(+)}(k \mathbf{r})= \\
& \frac{1}{k r}\left(\sqrt{n(n+1)} h_{n}^{(+)}(k r) \mathbf{Y}_{n, m}(\theta, \phi)+\xi_{n}^{(+)^{\prime}}(k r) \mathbf{Z}_{n, m}(\theta, \phi)\right),
\end{aligned}
$$

where $\mathbf{X}_{n, m}(\theta, \phi), \mathbf{Y}_{n, m}(\theta, \phi)$ and $\mathbf{Z}_{n, m}(\theta, \phi)$ are the Vector Spherical Harmonics [39], $k=\frac{2 \pi}{\lambda}, \lambda$ being the wavelength in the embedding medium and $\xi_{n}^{(+)^{\prime}}(k r)$ is the derivative with respect to $k r$. $n$ and $m$ are the total and projected angular momentum of the VPW, $n$ is classically called the multipolar order. $h_{n}^{(+)}(x)$ and $\xi_{n}^{(+)}(x)$ are respectively the spherical Hankel and the associated Ricatti-Hankel functions of the first kind. The regular VPWs are defined as follows:

$$
\begin{aligned}
& \mathbf{M}_{n, m}^{(1)}(k \mathbf{r})=j_{n}(k r) \mathbf{X}_{n, m}(\theta, \phi), \\
& \mathbf{N}_{n, m}^{(1)}(k \mathbf{r})= \\
& \frac{1}{k r}\left(\sqrt{n(n+1)} j_{n}(k r) \mathbf{Y}_{n, m}(\theta, \phi)+\psi_{n}^{\prime}(k r) \mathbf{Z}_{n, m}(\theta, \phi)\right),
\end{aligned}
$$

where $j_{n}(x)$ and $\psi_{n}(x)$ are respectively the spherical Bessel and associated Ricatti-Bessel functions. In the T-matrix framework, the total field outside the scatterer is split into the excitation and scattered field contributions. The expansions of the excitation and scattered electric fields 
read as follows:

$$
\begin{aligned}
& \mathbf{E}_{\mathrm{exc}}(k \mathbf{r})=E_{0} \sum_{n=1}^{\infty} \sum_{m=-n}^{n} e_{n, m}^{(h)} \mathbf{M}_{n, m}^{(1)}(k \mathbf{r})+e_{n, m}^{(e)} \mathbf{N}_{n, m}^{(1)}(k \mathbf{r}) \\
& \mathbf{E}_{\text {scat }}(k \mathbf{r})=E_{0} \sum_{n=1}^{\infty} \sum_{m=-n}^{n} f_{n, m}^{(h)} \mathbf{M}_{n, m}^{(+)}(k \mathbf{r})+f_{n, m}^{(e)} \mathbf{N}_{n, m}^{(+)}(k \mathbf{r})
\end{aligned}
$$

The internal field takes the following expression:

$$
\mathbf{E}_{\mathrm{int}}\left(k_{s} \mathbf{r}\right)=E_{0} \sum_{n=1}^{\infty} \sum_{m=-n}^{n} u_{n, m}^{(h)} \mathbf{M}_{n, m}^{(1)}\left(k_{s} \mathbf{r}\right)+u_{n, m}^{(e)} \mathbf{N}_{n, m}^{(1)}\left(k_{s} \mathbf{r}\right)
$$

where $k_{s}=n_{s} k$. The T-matrix coefficients are defined as follows $T_{n}^{(i)}(z)=\frac{f_{n, m}^{(i)}(z)}{e_{n, m}^{(i)}}$ where $i=e, h$ and $z=k R$. For spherical scatterers, the T-matrix coefficients can be computed using the continuity conditions leading to the following expression $T_{n}^{(i)}(z)=-\frac{N_{\mathrm{T}, n}^{(i)}(z)}{D_{n}^{(i)}(z)}$ in terms of the following numerator and denominator functions [33]:

$$
\begin{aligned}
& N_{\mathrm{T}, n}^{(e)}(z)=z \frac{\varepsilon_{s} j_{n}\left(n_{s} z\right) \psi_{n}^{\prime}(z)-\psi_{n}^{\prime}\left(n_{s} z\right) j_{n}(z)}{i n_{s}}, \\
& D_{n}^{(e)}(z)=z \frac{\varepsilon_{s} \xi_{n}^{(+)^{\prime}}(z) j_{n}\left(n_{s} z\right)-\psi_{n}^{\prime}\left(n_{s} z\right) h_{n}^{(+)}(z)}{i n_{s}}, \\
& N_{\mathrm{T}, n}^{(h)}(z)=-i z\left(\psi_{n}^{\prime}(z) j_{n}\left(n_{s} z\right)-\psi_{n}^{\prime}\left(n_{s} z\right) j_{n}(z)\right), \\
& D_{n}^{(h)}(z)=-i z\left(\xi_{n}^{(+)^{\prime}}(z) j_{n}\left(n_{s} z\right)-\psi_{n}^{\prime}\left(n_{s} z\right) h_{n}^{(+)}(z)\right),
\end{aligned}
$$

It is also necessary to introduce the $\Omega$-matrix coefficients linking the internal field to the incident field, $\Omega_{n}^{(i)}(z)=\frac{u_{n, m}^{(i)}(z)}{e_{n, m}^{(i)}}$. These coefficients can also be simply expressed by use of the denominator functions $\Omega_{n}^{(i)}(z)=\frac{1}{D_{n}^{(i)}(z)}$.

\subsection{Modal expansion of the scattered efficiency and internal field enhancements}

QNM analysis is common in wave physics [40,41] and has attracted a strong interest in photonics when studying resonant light interactions with nanocavities [34, 35, 42-49]. QNMs are the eigen-vector solutions of the Maxwell equations without sources and are associated with the complex eigenvalue frequencies $p_{n, \alpha}^{(i)}$ that also correspond to the poles of the scattering operators introduced in the previous section. For each multipolar order $n$, an infinite number of poles $\alpha$ exists. Poles associated with positive $\alpha$ have a positive real part while poles associated with negative $\alpha$ have a negative real part and are associated via the relation, $p_{n,-\alpha}=-p_{n, \alpha}^{*}$. In this study, we determine the eigen-frequencies $p_{n, \alpha}^{(i)}$ by calculating the poles of the scattering matrix. We use root-finding methods to locate the position of these poles starting from the asymptotic formulas of these poles provided in ref. [33]. We plot the real and imaginary parts of the first eigen-frequencies $\alpha=[-5 ; 5]$ of the electric dipole (Fig. 1a) and magnetic dipole (Fig. 1b) in the case of a spherical particle made of dielectric permittivity $\varepsilon_{s}=16$. The fundamental mode is $\alpha=0$ for the magnetic case and $\alpha= \pm 1$ for the electric case. It is characterized by a much larger leakage, i.e. a larger imaginary part in absolute value since poles in passive media have negative imaginary parts, than higher order modes. 
Following the derivation of the scattering operator by Grigoriev et al. [34], the modal expansions of the $T_{n}^{(i)}$ and $\Omega_{n}^{(i)}$ coefficients were recently derived [33,35]:

$$
\begin{aligned}
& T_{n}^{(i)}(z)=\frac{S_{\mathrm{nr}, n}^{(i)} e^{-2 i z}-1}{2}+\frac{e^{-2 i z}}{2} \sum_{\alpha=-M}^{+M} \frac{r_{n, \alpha}^{(i)}}{z-p_{n, \alpha}^{(i)}}, \\
& \Omega_{n}^{(i)}(z)=\frac{1}{2} \sum_{\alpha=-M}^{+M} \frac{r_{\Xi, n, \alpha}^{(i)}}{z-p_{n, \alpha}^{(i)}}
\end{aligned}
$$

where $r_{n, \alpha}^{(i)}$ is the residue of $T_{n}^{(i)}$ at the poles $p_{n, \alpha}^{(i)}, r_{\Xi, n, \alpha}^{(i)}$ is the residue of $\Omega_{n}^{(i)}(z)$ and $S_{\mathrm{nr}, n}^{(i)}=1+\sum_{\alpha=-M}^{+M} \frac{r_{n, \alpha}^{(i)}}{p_{n, \alpha}^{(i)}}$.

It can be shown that the convergence of this truncated series can be improved for a given truncation order $M$ by approximating the higher order tem $\alpha>M$ [35]. At the first order, the term that is removed when truncating the sum can be approximated by $-\sum_{\alpha=M+1}^{\infty} \frac{r_{n, \alpha}^{(i)}}{p_{n, \alpha}^{(i)}}-\sum_{\alpha=-(M+1)}^{-\infty} \frac{r_{n, \alpha}^{(i)}}{p_{n, \alpha}^{(i)}}$. This term is actually equal to $-\sum_{\alpha=M+1}^{\infty} \frac{r_{n, \alpha}^{(e)}}{p_{n, \alpha}^{(e)}}+\frac{r_{n, \alpha}^{(e)}}{p_{n,-\alpha}^{(e)}}=-\left((-1)^{n+1}-1\right)+\sum_{\alpha=-M}^{M} \frac{r_{n, \alpha}^{(e)}}{p_{n, \alpha}^{(e)}}$ and $-\sum_{\alpha=M+1}^{\infty} \frac{r_{n, \alpha}^{(h)}}{p_{n, \alpha}^{(h)}}+\frac{r_{n,-\alpha}^{(h)}}{p_{n,-\alpha}^{(h)}}=-\left((-1)^{n}-1\right)+\sum_{\alpha=-M}^{M} \frac{r_{n, \alpha}^{(h)}}{p_{n, \alpha}^{(h)}}$ [35]. The contribution from higher order poles $|\alpha|>M$ is then included in the term $S_{\mathrm{nr}, n}^{(i)}$ in order to improve the accuracy of the truncated expansion. The notation $A_{n}^{(i)}(z)=\frac{S_{\mathrm{nr}, n}^{(i)} e^{-2 i z}-1}{2}$ will be used in what follows.

Pole expansions in Eqs. (6) thus show the existence of a non-resonant term for the $T$-matrix coefficient only. The existence of a non-resonant contribution is also predicted by the Temporal Coupled Mode Theory (TCMT) [50-52]. However, TCMT is a phenomenological model that requires fitting parameters while Eqs. (6) derived here are free from fitting parameters and only rely on energy conservation principle $[34,35]$. This pole expansion permits us to derive the modal expansion of the multipolar scattering coefficients and therefore also the modal expansion of the scattering efficiency $Q_{\text {scat }, n}^{(i)}$ and internal field intensities averaged over the volume of the particle $\left\langle\left|E_{i n t, n}^{(i)}\right|^{2}\right\rangle$. The analytical expression of the residues for spherical scatterers can be obtained from the analytical expressions of the $\Omega$ and $T$ matrices [33,35]:

$$
r_{\Xi, n, \alpha}^{(e, h)}=\frac{1}{\left.\frac{d}{d z} D_{n}^{(e, h)}(z)\right|_{z=p_{n, \alpha}^{(e, h)}}}
$$

that yields the following expression for $r_{\Xi, n, \alpha}^{(e, h)}$ :

$$
\begin{aligned}
& r_{\Xi, n, \alpha}^{(e)}=\frac{1}{\varepsilon_{s}-1} \times \\
& \frac{2 i n_{s}}{\xi_{n}^{(+)^{\prime}}\left(p_{n, \alpha}^{(e)}\right) \psi_{n}^{\prime}\left(n_{s} p_{n, \alpha}^{(e)}\right)+n(n+1) h_{n}^{(+)}\left(p_{n, \alpha}^{(e)}\right) j_{n}\left(n_{s} p_{n, \alpha}^{(e)}\right)} \\
& r_{\Xi, n, \alpha}^{(h)}=\frac{2 i}{\left(\varepsilon_{s}-1\right)\left(p_{\alpha}^{(h)}\right)^{2} j_{n}\left(n_{s} p_{\alpha}^{(h)}\right) h_{n}^{(+)}\left(p_{\alpha}^{(h)}\right)}
\end{aligned}
$$

where the following identities of the Ricatti-Hankel and Ricatti-Bessel functions have been used: $\xi_{n}^{(+)^{\prime \prime}}(z)=\frac{n(n+1)-z^{2}}{z} h_{n}^{(+)}(z)$ and $\psi_{n}^{(+)^{\prime \prime}}(z)=\frac{n(n+1)-z^{2}}{z} j_{n}(z) . r_{n, \alpha}^{(e, h)}$ can also be analytically determined for spherically-symmetric scatterers from: 

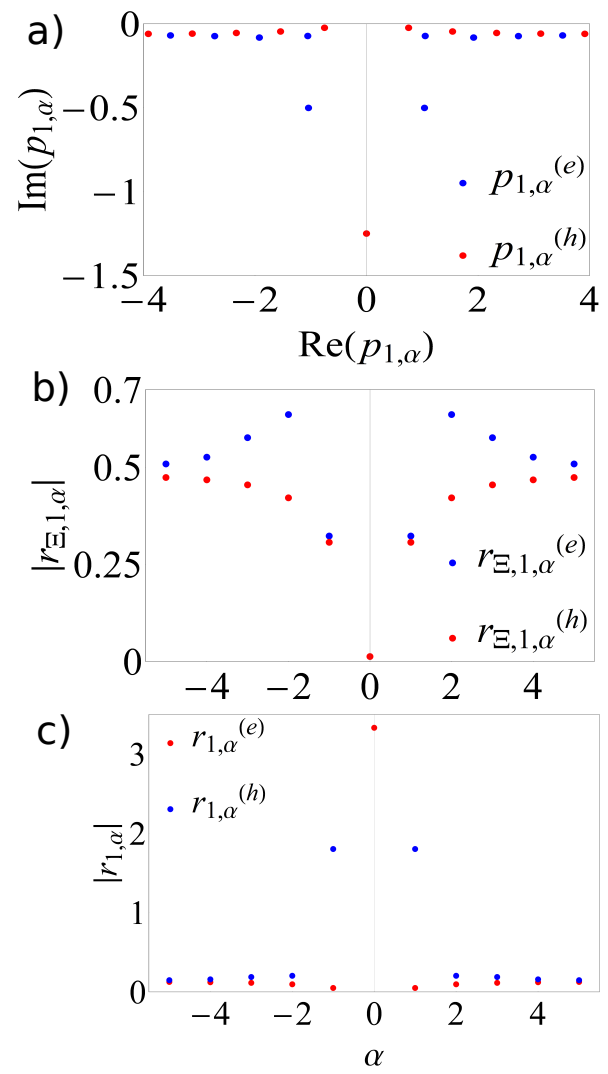

Fig. 1. a) Position of the first poles of the dipolar electric and magnetic scattering coefficients in the complex frequency plane. b) Modulus of the associated residues of $T_{n}^{(h)}:\left|r_{1, \alpha}^{(e)}\right|$ and $\left|r_{1, \alpha}^{(h)}\right|$. c) Modulus of the associated residues of $\Omega_{n}^{(h)}:\left|r_{\Xi, 1, \alpha}^{(e)}\right|$ and $\left|r_{\Xi, 1, \alpha}^{(h)}\right|$.

$$
r_{n, \alpha}^{(e, h)}=-\frac{e^{2 i p_{n, \alpha}^{(e, h)}} N_{n}^{(e, h)}\left(p_{n, \alpha}^{(e, h)}\right)}{\left.\frac{d}{d z} D_{n}^{(e, h)}(z)\right|_{z=p_{n, \alpha}^{(e, h)}}}
$$

The set of $r_{n, \alpha}^{(e, h)}$ consequently takes the following expression:

$$
\begin{aligned}
& r_{n, \alpha}^{(e)}=\frac{j_{n}\left(n_{s} p_{n, \alpha}^{(e)}\right)}{h_{n}^{(+)}\left(p_{n, \alpha}^{(e)}\right)} \frac{1}{\varepsilon_{s}-1} \times \\
& \frac{2 i \varepsilon_{s} e^{2 i p_{n, \alpha}^{(e)}}}{\xi_{n}^{(+)^{\prime}}\left(p_{n, \alpha}^{(e)}\right) \psi_{n}^{\prime}\left(n_{s} p_{n, \alpha}^{(e)}\right)+n(n+1) h_{n}^{(+)}\left(p_{n, \alpha}^{(e)}\right) j_{n}\left(n_{s} p_{n, \alpha}^{(e)}\right)} \\
& r_{n, \alpha}^{(h)}=\frac{j_{n}\left(n_{s} p_{n, \alpha}^{(h)}\right)}{h_{n}^{(+)}\left(p_{n, \alpha}^{(h)}\right)} \frac{2 i e^{2 i p_{n, \alpha}^{(h)}}}{\left(\varepsilon_{s}-1\right)\left(p_{n, \alpha}^{(h)}\right)^{2} j_{n}\left(n_{s} p_{n, \alpha}^{(h)}\right) h_{n}^{(+)}\left(p_{n, \alpha}^{(h)}\right)}
\end{aligned}
$$

The scattering efficiency $Q_{\text {scat }}$ of a scatterer can be calculated with respect to the electric 
and magnetic scattering Mie coefficients $a_{n}$ and $b_{n}$ that are simply the opposite of the $T$-matrix coefficients $a_{n}=-T_{n}^{(e)}$ and $b_{n}=-T_{n}^{(h)}$ :

$$
\begin{aligned}
& Q_{\text {scat }, n}^{(e)}=\frac{2(2 n+1)}{z^{2}}\left|a_{n}\right|^{2}=\frac{2(2 n+1)}{z^{2}}\left|T_{n}^{(e)}\right|^{2} \\
& Q_{\text {scat }, n}^{(h)}=\frac{2(2 n+1)}{z^{2}}\left|b_{n}\right|^{2}=\frac{2(2 n+1)}{z^{2}}\left|T_{n}^{(h)}\right|^{2}
\end{aligned}
$$

A similar approach can be followed with the internal Mie coefficients $d_{n}$ and $c_{n}$ that can be derived from the $\Omega$ matrix elements: $d_{n}=\Omega_{n}^{(e)}, c_{n}=\Omega_{n}^{(h)}$. The average of the electric field intensity over the volume inside the spherical particle $\left\langle\left|E_{\mathrm{int}}\right|^{2}\right\rangle$ can be calculated with respect to the multipolar components of the electric field $E_{\text {int }, n}^{(i)}[33]$ :

$$
\left\langle\left|E_{\mathrm{int}}\right|^{2}\right\rangle=\sum_{n}\left\langle\left|E_{\mathrm{int}, n}^{(e)}\right|^{2}\right\rangle+\left\langle\left|E_{\mathrm{int}, n}^{(h)}\right|^{2}\right\rangle
$$

where :

$$
\begin{aligned}
& \frac{\left\langle\left|\mathbf{E}_{i, n}^{(e)}\right|^{2}\right\rangle}{\left|\mathbf{E}_{0}\right|^{2}}=\frac{3(2 n+1)}{2 z^{2}} I_{n}\left(\varepsilon_{s}, z\right)\left|\Omega_{n}^{(e)}\right|^{2}, \\
& \frac{\left\langle\left|\mathbf{E}_{i, n}^{(h)}\right|^{2}\right\rangle}{\left|\mathbf{E}_{0}\right|^{2}}=\frac{3(2 n+1)}{2 z^{2}} J_{n}\left(\varepsilon_{s}, z\right)\left|\Omega_{n}^{(h)}\right|^{2},
\end{aligned}
$$

with:

$$
\begin{aligned}
& I_{n}\left(\varepsilon_{s}, z\right)=\frac{z}{n_{s}^{2}} \times \\
& \psi_{n}^{\prime}\left(n_{s} z\right)\left(\psi_{n}^{\prime}\left(n_{s} z\right)+j_{n}\left(n_{s} z\right)\right)+\left(\left(n_{s} z\right)^{2}-n(n+1)\right) j_{n}^{2}\left(n_{s} z\right) \\
& J_{n}\left(\varepsilon_{s}, z\right)=\frac{z}{n_{s}^{2}} \times \\
& \psi_{n}^{\prime}\left(n_{s} z\right)\left(\psi_{n}^{\prime}\left(n_{s} z\right)-j_{n}\left(n_{s} z\right)\right)+\left(\left(n_{s} z\right)^{2}-n(n+1)\right) j_{n}^{2}\left(n_{s} z\right) .
\end{aligned}
$$

The modal expansion of the internal field enhancement in the whole scatterer allows to determine the mode of each multipolar order that yields the strongest field enhancement inside the particle. Combined with the modal expressions of the scattered efficiency (Eq. 9), we can compare the spectra of the internal and external fields and analyze the origin of the scattering anomalies in terms of QNMs.

\section{Results and discussions}

Let us first compare the spectra of the averaged field enhancement with the scattering efficiencies in the case of a spherical particle of dielectric permittivity $\varepsilon=16$, value closed to the dielectric permittivity of silicon in visible and near infrared spectrum (see Fig. 2). For that purpose, we plot in Fig. 2(a-b) the scattering efficiency associated with the electric $Q_{\text {scat }, 1}^{(e)}$ and magnetic $Q_{\text {scat }, 1}^{(h)}$ dipolar contributions, and of the averaged field intensity inside the particle $\left\langle\left|E_{i n t, 1}^{(i)}\right|^{2}\right\rangle$, with respect to the size parameter $z=k R$ when the particle is illuminated by a plane wave. $Q_{\text {scat }, 1}^{(i)}$ and $\left\langle\left|E_{i n t, 1}^{(i)}\right|^{2}\right\rangle$ both exhibit a resonant behavior with the presence of several peaks when increasing the parameter size $z$. These plots are obtained using conventional multipolar formalism and 

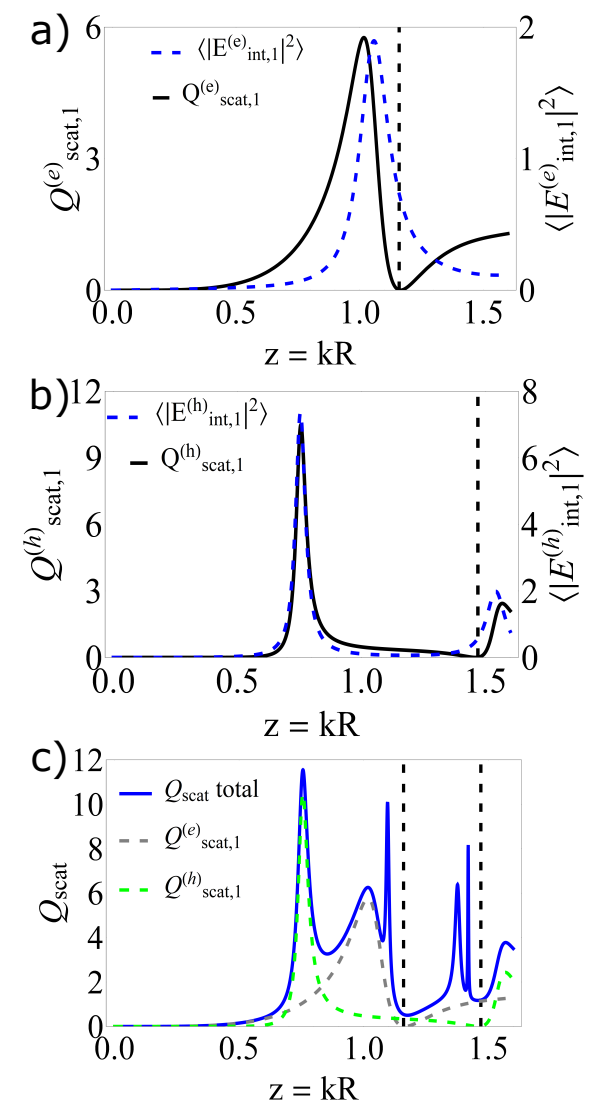

Fig. 2. (a,b) Multipolar partial scattering efficiencies versus internal field enhancement. Left scale, black full line : dipolar scattering efficiencies calculated with Eqs. 9, right scale, blue dashed line: averaged internal field intensity $\left\langle\left|E_{\text {int }, 1}\right|^{2}\right\rangle$ calculated with Eqs. 10 with respect to the parameter size $z$. Vertical dashed lines indicate the minimum of the scattering efficiency. (a) Electric dipole, (b) Magnetic dipole. (c) Scattering spectrum. Blue line: total scattering (with $\mathrm{N}=10$ ); grey dashed line: electric dipole $(\mathrm{N}=1, \mathrm{e})$; green dashed line: magnetic dipole $(\mathrm{N}=1, \mathrm{~h})$.

will be compared later in Figs. 3,4 with results calculated with our modal expansion. As can be seen in Fig. 2(a-b), the peaks associated with $Q_{s c a t, 1}^{(i)}$ and $\left\langle\left|E_{i n t, 1}^{(i)}\right|^{2}\right\rangle$, for both the electric $i=e$ (Fig. 2a) and magnetic $i=h$ (Fig. 2b) cases occur at nearby frequencies. A careful analysis reveals that the peak frequencies are slightly different for the electric case, while they are almost equal for the magnetic case.

A more fundamental difference can be noticed when observing the shape of the resonances: while $\left\langle\left|E_{\text {int }, 1}^{(e)}\right|^{2}\right\rangle$ and $\left\langle\left|E_{\text {int }, 1}^{(h)}\right|^{2}\right\rangle$ feature pure Lorentzian shapes, the scattering efficiencies $Q_{\text {scat }, 1}^{(i)}$ $(i=e, h)$ feature resonances with asymmetric line shapes referred as Fano resonances [6, 53-59]. In particular, for the electric case, the maximum of $Q_{s c a t, 1}^{(e)}$ is closely followed by a minimum of the scattering efficiency. For the magnetic case, the first peak at $z \approx 0.75$ features an almost perfect Lorentzian shape while the second peak at $z \approx 1.5$ is associated with a strong minimum.

As the different multipolar contributions are well separated, these minima of $Q_{s c a t, 1}^{(e)}$ and $Q_{\text {scat }, 1}^{(h)}$ correspond to low total scattered fields as can be seen in Fig. 2(c). Importantly, position 

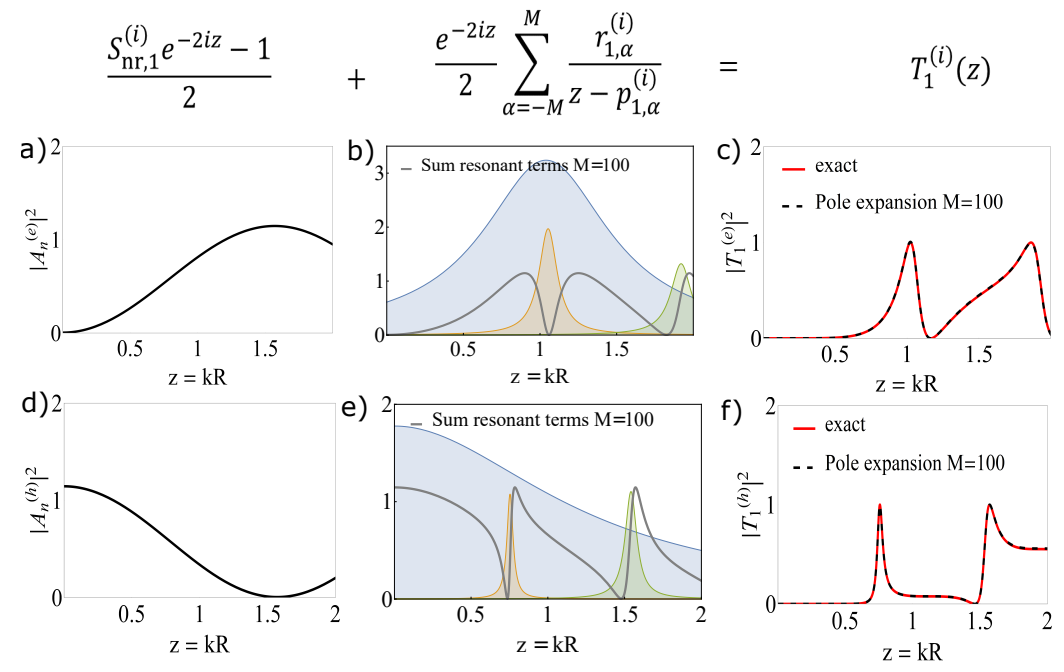

Fig. 3. Analysis of the different terms in the modal expansion of the $T_{1}^{(i)}(z)$-matrix element. $(\mathrm{a}, \mathrm{d})$ : Non-resonant term $A_{1}^{(i)}(z)=\frac{S_{\mathrm{nr}, 1}^{(i)} e^{-2 i z}-1}{2}$ with $i=e(\mathrm{a})$ and $i=h(\mathrm{~d})$; (b,e): Resonant term $\left|\frac{e^{-2 i z}}{2} \frac{r_{1, \alpha}^{(i)}}{z-p_{1, \alpha}^{(i)}}\right|^{2}$ with: blue line+shadow $\alpha=1$ (b) and $\alpha=0$ (e), orange line+shadow $\alpha=2$ (b) and $\alpha=1$ (e), green line+shadow $\alpha=3$ (b) $\alpha=2$ (e); Grey line: resonant term with summation over poles: $\frac{e^{-2 i z}}{2} \sum_{\alpha=-M}^{M} \frac{r_{\alpha, 1}^{(e)}}{z-p_{\alpha, 1}^{(e)}}$ with $M=100$. (c,f): non-resonant + resonant terms with summation and $M=100$ (complete pole expansion of the $T_{1}^{(i)}(z)$-matrix element): black dashed line, full calculations: red full line.

of the anapole condition, corresponding to a minimum of $Q_{s c a t, 1}^{(e)}$ and $Q_{s c a t, 1}^{(h)}$ and indicated by vertical dashed lines in Fig. 2(c), can be well predicted when considering only dipolar contributions. The minimum is more pronounced for the electric dipole coefficient than for the magnetic dipole due to the presence of non-negligible contributions from higher order modes. The higher order modes are at the origin of the narrow peaks observed in Fig. 2c between $z=[1 ; 1.5]$. It is also observed that at these minimum frequencies, the average field intensity remains enhanced by the resonances which means that, at certain frequencies, the scattering efficiency can be strongly minimized while the internal field is enhanced by a resonant behavior of the internal scattered field coefficients: this condition is commonly referred as an anapole condition $[11,21,23-25]$.

The different terms of the modal expansion of $T_{1}^{(e)}(z)$ (top line) and $T_{1}^{(h)}(z)$ (bottom line) (Eq. 6) are plotted in Fig. 3. Two types of terms can be distinguished: there is $(i)$ a non-resonant term associated with $A_{n}^{(i)}(z)=\frac{S_{\mathrm{nr}, n}^{(i)} e^{-2 i z}-1}{2}$ that corresponds to a slowly varying background (see Fig. 3a,d) [34,57] and (ii) a resonant term $\frac{e^{-2 i z}}{2} \sum_{\alpha=-M}^{M} \frac{r_{n, \alpha}^{(i)}}{z-p_{n, \alpha}^{(i)}}$ associated with the poles $p_{n, \alpha}^{(i)}$ (see Fig. 3b,e). The plot of the non-resonant term in Fig. 3(a,d) highlights the $\pi$ phase shift between the electric and magnetic cases: while this non-resonant term is maximum at $z=0$ and null at $z=1.5$ for the magnetic case, it is null at $z=0$ and maximum at $z=1.5$ for the electric case. Therefore the non-resonant contribution plays a larger role for the electric low order resonances than for the magnetic ones.

Let us now investigate the role of the resonant terms by plotting in Fig. 3(b,e) the contributions 
of the first three modes separately: $\alpha=[1 ; 3]$ for the electric case and $\alpha=[0 ; 2]$ for the magnetic case. In all cases, it can be seen that they correspond to pure Lorentzian shapes. For both electric and magnetic cases, the first mode ( $\alpha=1$ for electric, $\alpha=0$ for the magnetic) has a very poor quality factor which means that it overlaps with the higher order modes. An important question arises now: can a minimum of the far-field spectrum arise when taking into account the sum of the mode contributions only, i.e. without taking into account the $A_{1}^{(i)}(z)$ term? To answer this question, we plot in grey line the resonant term $\left|\frac{e^{-2 i z}}{2} \sum_{\alpha=-M}^{+M} \frac{r_{1, \alpha}^{(i)}}{z-p_{1, \alpha}^{(i)}}\right|^{2}$ in Fig. 4b,e (see Eq.6) while considering a large number of poles (e.g. $M=100)$, but neglecting the non-resonant term $A_{1}^{(i)}(z)$. The sum of the different pole contributions does not lead to Lorentzian responses but to asymmetric Fano-like shapes featuring strong minima. This result is a direct consequence of the fact that the different modes interfere. In fact, the QNM are not orthogonal in the sense of the usual conjugated scalar product. This means that energy can be exchanged between different modes leading to a phenomenon of interference. That is the reason why interference between modes may appear in the scattering efficiency spectrum. However, let us point out that an unconjugated scalar product can also be defined for which QNM become orthogonal even though they can exchange energy $[46,49,60]$. In Fig. 3b, we see that, in the electric case, the first minimum of $\left|\frac{e^{-2 i z}}{2} \sum_{\alpha=-M}^{+M} \frac{r_{1, \alpha}^{(e)}}{z-p_{1, \alpha}^{(e)}}\right|^{2}$ occurs when the modes $\alpha=1$ and $\alpha=2$ that have the same real frequency are in phase opposition. The difference of phase between the modes involved in the destructive interference are plotted in Fig. 4e-f, i.e. $\phi_{1}-\phi_{2}$ in the electric case and $\phi_{0}-\phi_{2}$ in the magnetic case where $\phi_{\alpha}=\operatorname{Arg}\left[\frac{e^{-2 i z}}{2} \frac{r_{1, \alpha}^{(i)}}{z-p_{1, \alpha}^{(i)}}\right]$. We can see in Fig. 4e-f that the difference of phase turns out to be equal to $\pi$ near the destructive interference between the modes. For these two modes to destructively interfere, not only should they be in phase opposition but they also need to have the same amplitude. The amplitude is quantified by the expression $\left|\frac{r_{\alpha, n}^{(i)}}{z-p_{\alpha, n}^{(i)}}\right|^{2}$, which shows that a resonant contribution is proportional to the residue $r_{\alpha, n}^{(i)}$ and requires poles $p_{\alpha, n}^{(i)}$ with small imaginary parts to maximize $\frac{1}{z-p_{\alpha, n}^{(i)}}$. Here the pole $\alpha=1$ is characterized by a much larger imaginary part than the pole $\alpha=2$ but this weak quality factor is compensated by a larger residue $r_{\alpha=1,1}^{(e)}$ leading to $\left|\frac{r_{1,1}^{(e)}}{z-p_{1,1}^{(e)}}\right| \approx\left|\frac{r_{1,2}^{(e)}}{z-p_{1,2}^{(e)}}\right|$ (residues are plotted with respect to $\alpha$ in Fig.1). This condition is fulfilled near $\mathfrak{R}\left[p_{1,1}^{(e)}\right] \approx \mathfrak{R}\left[p_{1,2}^{(e)}\right]$.

In the electric case, the sum of the resonant terms $\left|\sum r_{1, \alpha}^{(h)} /\left(z-p_{1, \alpha}\right)^{(h)}\right|$ displayed in Fig. 3(e) shows that a minimum is associated with both $\alpha=1$ and $\alpha=2$ modes since they both interfere destructively with the low quality $\alpha=0$ mode. Minima always occur at the vicinity of the real part of one pole, $\mathfrak{R}\left[p_{1, \alpha}^{(i)}\right]$, since the function $\frac{e^{-2 i z}}{2} \frac{r_{1, \alpha}^{(i)}}{z-p_{1, \alpha}^{(i)}}$ experiences an abrupt change of phase. The resonant contribution from this mode can thus become out of phase with the contribution of the low quality mode. A similar explanation holds for the appearance of minimum scattering in the magnetic case: it results from the destructive interference between the wide resonance associated with pole $\alpha=0$ with a narrow resonance associated with poles $\alpha=1$ and $\alpha=2$. However, in this case, the first minimum close to the pole $\alpha=1$ disappears when the non-resonant contribution is taken into account. Moreover, concerning the minimum associated with $\alpha=2$, the shift due to the non-resonant contribution is minor as $A_{1}^{(h)}(z)$ is almost null in the vicinity of the peak due to the pole $\alpha=2$ (see Fig.3d).

At this stage, it has been established that minima in the scattering spectra result from the interference between a low and a high quality factor mode. We now study the influence of the non-resonant contribution on the spectral location of such minima. For that purpose, the spectrum 

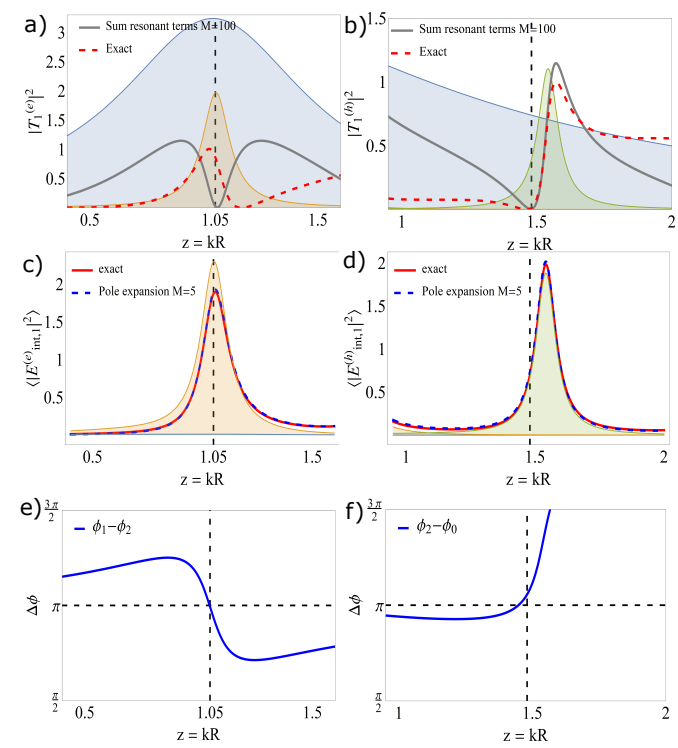

Fig. 4. Spectra of the average internal field compared with the spectra of the scattering efficiencies for the magnetic and electric cases. (a) and (b) are taken from Fig. 3(b,c,e,f). Grey line: resonant contribution only in Eq. 6 with $M=100$, red dotted line: resonant + non resonant contributions with $M=100$. (c) and (d): Square of the norm of the internal electric field averaged over the volume of the particle $\left\langle\left|E_{i n t, n}^{(i)}\right|^{2}>, i=e\right.$ for (c) and $i=h$ for (d). Orange and green lines+shadow: modal expansion calculated with Eqs.10-11 when taking into account a single pole: $\alpha=2$ (orange), $\alpha=3$ (green) or when summing $2 M$ poles (blue dashed line with $M=5$ ). Full red line: full calculations. The vertical dashed lines indicate the spectral position of the anapole condition. (e) and (f): Difference of phase between the modes $\phi_{1}-\phi_{2}$ in the electric case and $\phi_{0}-\phi_{2}$ in the magnetic case with $\phi_{\alpha}=\operatorname{Arg}\left[\frac{e^{-2 i z}}{2} \frac{r_{1, \alpha}^{(i)}}{z-p_{1, \alpha}^{(i)}}\right]$.

of the resonant contribution only is compared with exact calculations and modal calculations that include both resonant and non-resonant terms in Fig. 3(c,f). A spectral shift between these spectra with or without non-resonant contribution can be observed, especially for the electric case. The spectral shift is less pronounced for the magnetic case (Fig. 3(f)) as the magnetic non-resonant term $A_{n}^{(h)}(z)$ is almost null when $z \approx 1.6$, i.e. close to the frequency where the modes destructively interfere (Fig. 3(c)). In all cases, it is necessary to add up the non-resonant contribution $A_{n}^{(i)}(z)$ in order to accurately retrieve the position of the peaks and dips of $\left|T_{1}^{(i)}\right|^{2}$. Let us now explain why the first resonance of $\left|T_{1}^{(h)}\right|$ at $z \approx 0.75$ is not associated with an anapole. Indeed, a strong minimum is observed near $\mathfrak{R}\left[p_{1,2}^{(h)}\right]$ when the resonant contributions only are summed. This minimum completely disappears when the non-resonant contribution is included as shown in Fig. 3(f). This shows that the non-resonant contribution destructively interferes with the contribution from $\alpha=0$. For the first peak of $\left|T_{1}^{(h)}\right|^{2}$, the contribution from the mode $\alpha=1$ is predominant and a peak with almost a Lorentzian shape is observed in Fig. 3(f). In Fig. 3(c,f), it is shown that a near perfect agreement with exact calculations (Mie theory) is found while taking into account both resonant $(M=100)$ and non-resonant contributions. Minima of the scattering spectrum therefore result not only from $(i)$ the interference between the mode contributions (the eigenfields associated with complex frequencies are not orthogonal) but also 
from (ii) the presence of a non-resonant term.

The internal field can also be studied in the framework of the modal expansion in Eqs. 10-11. The accuracy and the convergence of this pole expansion of the internal field are illustrated in Fig. 4 where the exact value of $\left\langle\left|E_{i n t, 1}^{(i)}\right|^{2}\right\rangle$ is compared to the pole expansions in Eqs. 10-11 when considering one or two poles, i.e. $M=5$ (top line: electric case $(i=e)$; bottom line: magnetic case $(i=h)$ ). Each of the Lorentzian-type resonances appearing in the spectrum of the internal field is associated with one complex resonance frequency. Remarkably, it can be observed that the modal expansion fits perfectly the rigorous calculations with a very limited number of poles. While a large number of poles was required to match the rigorous calculations of the scattered field, the convergence is much more rapidly achieved in the case of the internal field.

In Fig. 4(a,c), we focus on the first resonance of the electric dipolar scattering coefficient that exhibits an anapole behavior. The explanation of the dip in the spectrum of the scattering coefficient was earlier explained at length. Just as in the electric case, the resonance of the internal field is mostly due to the resonant contribution associated with the mode $\alpha=2$. The mode $\alpha=1$ only contributes weakly to the internal field enhancement; its contribution is too weak to be visible in Fig. 4 whereas it played a key role in the appearance of a dip in the scattering coefficient spectrum. This is the main difference between external and internal fields: the fundamental modes, $\alpha=0$ in the magnetic case and $\alpha=1$ in the electric case, contribute only for the external field, and not for the internal field. This is due to the fact that the residues of the fundamental modes in the internal fields are much smaller than those of higher order modes. The first poles of the dipolar electric Mie coefficients and the modulus of the associated residues $r_{\Xi, 1, \alpha}^{(i)}$ and $r_{1, \alpha}^{(i)}$ for an $\varepsilon=16$ particle in air are plotted in Fig 1 .

As the quality factor associated with the fundamental mode is also much weaker than that of higher order modes (see Fig.1), we get the relation: $\frac{r_{\Xi, 1, \alpha=1}^{(e)}}{z-p_{1, \alpha=1}^{(e)}} \ll \frac{r_{\Xi, 1, \alpha>1}^{(e)}}{z-p_{1, \alpha>1}^{(e)}}$ and $\frac{r_{\Xi, 1, \alpha=0}^{(h)}}{z-p_{1, \alpha=0}^{(h)}} \ll \frac{r_{\Xi, 1, \alpha>0}^{(h)}}{z-p_{1, \alpha>0}^{(h)}}$. Physically, this means that in the external field, the fundamental mode with low quality factor can therefore interfere with the higher order modes of higher quality factor leading to Fano resonances, while in the internal field, this fundamental mode does not interfere with higher order modes and the averaged internal field only exhibits Lorentzian-like resonances driven by higher order modes that are spectrally well separated. Fig. 4(a,c) also show that the position of the maximum internal field enhancement would correspond to the position of the dip of the scattering coefficients without the additional blue-shift due to the non-resonant term. However, this minimum shifts when the non-resonant contribution is added so that the minimum of the scattered field does not correspond in general to the maximum of the internal field.

In Fig. 4(b,d), we focus on the second resonance of $T_{1}^{(h)}$. We do not concentrate on the first resonance since it does not exhibit an anapole behavior as explained earlier. The mode $\alpha=0$ that interfered with the mode $\alpha=2$ to yield the dip in the spectrum of $T_{1}^{(h)}(z)$ contributes only weakly to the internal field. The resonance of the averaged internal field is then almost entirely due to the mode $\alpha=2$ and thus possesses a Lorentzian shape.

The distributions of the electric field intensity in the dielectric Mie scatterer are plotted in Fig. 5 at frequencies that maximize the average internal field intensity $(z=1.065)$ and minimize the scattering efficiency $(z=1.160)$. The field distributions are significantly different and we remark $(i)$ the anapole-like field distribution at the minimum of the scattering efficiency and (ii) the stronger field enhancement at a frequency different from the anapole frequency. These results highlight the fact that anapoles do not necessarily lead to an optimal internal field enhancement in average inside the scatterer. Also, when comparing the averaged field intensity enhancement between the electric dipole, electric anapole and magnetic dipole, it is clear that the maximum of field enhancement is achieved for the first magnetic dipolar mode (see also Fig. 2).

After having thoroughly analyzed the anapole conditions for the important case of $\varepsilon=16$, 

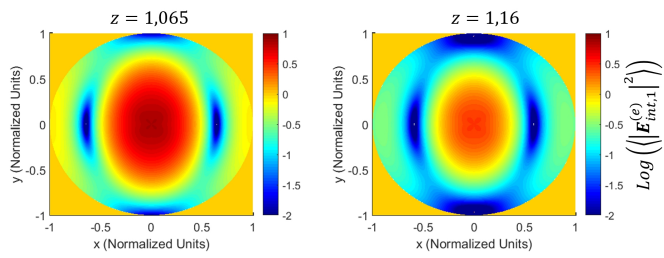

Fig. 5. Internal field enhancement $\log \left|E_{i n t, 1}^{(e)}\right|^{2}$ in the plane containing the incident electric field plotted inside the spherical scatterer at the electric dipole resonance, $z=\mathfrak{R}\left(p_{1, \alpha}^{(i)}\right)=$ 1.065 (left), and at the anapole condition $z=1.160$ (right). The field enhancement is stronger at the electric dipole resonance (left).
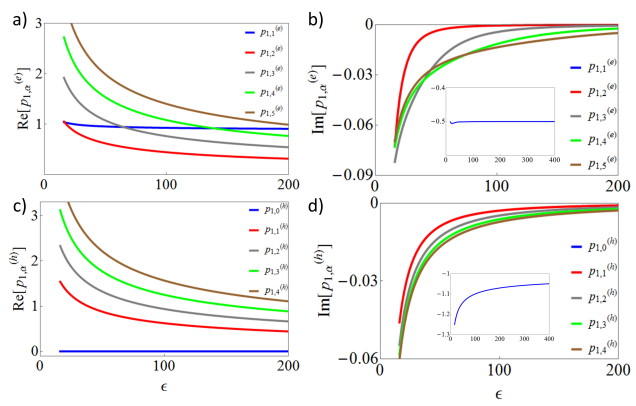

Fig. 6. Trajectories of the 5 first dipolar eigen-frequencies in the complex frequency plane when increasing the dielectric permittivity $\varepsilon$ of the scatterer. Real and imaginary parts of the eigen-frequencies $p_{1, \alpha}^{(i)}$ of the electric $(i=e, \mathrm{a}$ and $\mathrm{b})$ and magnetic $(i=h, \mathrm{c}$ and d) dipolar modes $n=1$ with respect to the dielectric permittivity of the scatterer embedded in air. The 5 first dipolar modes are plotted: $\alpha=[1 ; 5]$ for the electric case (a and b); $\alpha=[0 ; 4]$ for the magnetic case (c and d). The imaginary part of the first mode ( $\alpha=0$ for $i=(h)$ and $\alpha=1$ for $i=(e))$ is plotted in inset.

one now aims at generalizing this analysis for arbitrary dielectric permittivities. As anapole conditions can be predicted by calculating the poles of the resonant scatterer, the trajectories of the first 5 poles of the electric and magnetic dipolar Mie coefficients $p_{1, \alpha}^{(e)}, p_{1, \alpha}^{(h)}, \alpha=[1 ; 5]$ are plotted in the complex frequency planes when increasing the dielectric permittivity of the resonant scatterer. For both magnetic and electric cases, it turns out that the real and imaginary parts of the eigen-frequencies $p_{1, \alpha}$ of the first poles ( $\alpha=0$ for the magnetic case and $\alpha=1$ for the electric case) are almost insensitive to the dielectric permittivity and that the imaginary part is much higher in magnitude than all the other poles. $p_{1,0}^{(h)}$ features the peculiarity of having a real part equal to 0 . On the other hand, all the other poles depend strongly on the dielectric permittivity of the scatterer. Their real parts decrease, meaning that resonances occur for smaller size parameters $z$, and their imaginary parts decrease in magnitude and tend toward zero. The high quality factor of the higher order modes will prohibit any significant interference effect between them but the low quality factor of the fundamental mode leads to interference effects with higher order modes. The anapole location on the spectrum is therefore driven by the high quality factor resonance, i.e. the scattering spectrum features sharp minima close to the real parts of the high quality modes. For the electric case, the blue-shift of the anapole due to the non-resonant term decreases with increasing $\varepsilon$ since the non-resonant term $A_{1}^{(e)}(z) \rightarrow 0$ when $z \rightarrow 0$. The 
contrary happens for the magnetic mode since the electric and magnetic non-resonant terms are in phase opposition as illustrated in Fig.3(a,d). Regarding the internal field, it should be first noticed that the internal field enhancement depends on the quality factor that describes the ability of the cavity to trap light. Therefore, the internal field associated with the fundamental modes characterized by large values of $\mathfrak{I}\left(p_{n, \alpha}^{(i)}\right)$ do not lead to significant internal field enhancements. As all the higher order modes ( $\alpha>0$ for the magnetic case and $\alpha>1$ for the electric case) do not overlap due to their narrowness, and due to the absence of a non-resonant term for the internal coefficients, the averaged field inside the scatterer features almost pure Lorentzian shapes.

\section{Conclusion}

To conclude, anapoles in Mie resonators result from the existence of electric and magnetic fundamental modes characterized with a weak quality factor that interfere with higher order modes associated with much higher quality factors. The interference is also affected by a non-resonant term that can shift the spectral position of the scattering minimum. The destructive interference leads to a Fano resonance associated with a minimum of the scattering efficiency. This modal analysis also clarifies the link between Fano resonances, anapole conditions and internal field enhancements. We used a modal expansion of the light intensity enhancement over the whole volume of the scatterer to identify the conditions that yield the maximum of the internal field enhancement in spherical Mie scatterers. The maximum of the field enhancement does not occur at the anapole condition but at the real part of the eigen-frequencies of high quality factor modes. The field inside the scatterer does not feature any minimum since it is only weakly affected by the fundamental modes with low quality factor and the modal expansion of the internal field coefficients does not possess a non-resonant term. Consequently, the internal field spectrum shows peaks associated with eigenmodes (except the fundamental low $Q$ mode) and features almost pure Lorentzian shapes. This also means that the internal field associated with an anapole signature in the far field does not yield a particular field confinement inside the scatterer since it results simply from the excitation of a given mode in the dielectric particle. This modal analysis is carried out in the framework of a multipolar expansion of the field and could be further extended to non-spherical objects using appropriate multipolar decompositions.

\section{Acknowledgments}

Research conducted within the context of the International Associated Laboratory ALPhFA: Associated Laboratory for Photonics between France and Australia. This work has been carried out thanks to the support of the A*MIDEX project (no. ANR-11-IDEX-0001-02) funded by the Investissements d'Avenir French Government program, managed by the French National Research Agency (ANR). We acknowledge support by the Australian Research Council.

\section{References}

1. A. F. Koenderink, A. Alù, and A. Polman, "Nanophotonics: Shrinking light-based technology," Science 348, 516-521 (2015).

2. A. I. Kuznetsov, A. E. Miroshnichenko, M. L. Brongersma, Y. S. Kivshar, and B. Luk'yanchuk, "Optically resonant dielectric nanostructures," Science 354, aag2472 (2016).

3. A. Krasnok, M. Caldarola, N. Bonod, and A. Alú, "Spectroscopy and biosensing with optically resonant dielectric nanostructures," Adv. Opt. Mater. 6, 1701094 (2018).

4. A. Devilez, X. Zambrana-Puyalto, B. Stout, and N. Bonod, "Mimicking localized surface plasmons with dielectric particles," Phys. Rev. B 92, 241412 (2015).

5. M. R. Shcherbakov, D. N. Neshev, B. Hopkins, A. S. Shorokhov, I. Staude, E. V. Melik-Gaykazyan, M. Decker, A. A. Ezhov, A. E. Miroshnichenko, I. Brener, A. A. Fedyanin, and Y. S. Kivshar, "Enhanced third-harmonic generation in silicon nanoparticles driven by magnetic response," Nano Lett. 14, 6488-6492 (2014).

6. M. I. Tribelsky and A. E. Miroshnichenko, "Giant in-particle field concentration and fano resonances at light scattering by high-refractive-index particles," Phys. Rev. A 93, 053837 (2016). 
7. V. Gili, L. Carletti, A. Locatelli, D. Rocco, M. Finazzi, L. Ghirardini, I. Favero, C. Gomez, A. Lemaître, M. Celebrano, C. De Angelis, and G. Leo, "Monolithic algaas second-harmonic nanoantennas," Opt. Express 24, 15965-15971 (2016).

8. P. Kapitanova, V. Ternovski, A. Miroshnichenko, N. Pavlov, P. Belov, Y. Kivshar, and M. Tribelsky, "Giant field enhancement in high-index dielectric subwavelength particles," Sci. Rep. 7, 731 (2017).

9. R. Colom, L. Xu, L. Marini, F. Bedu, I. Ozerov, T. Begou, J. Lumeau, A. E. Miroshnichenko, D. N. Neshev, B. Kuhlmey, S. Palomba, and N. Bonod, "Enhanced four-wave mixing in doubly resonant si nanoresonators," ACS Photonics 6, 1295-1301 (2019).

10. M. R. Shcherbakov, P. P. Vabishchevich, A. S. Shorokhov, K. E. Chong, D.-Y. Choi, I. Staude, A. E. Miroshnichenko, D. N. Neshev, A. A. Fedyanin, and Y. S. Kivshar, "Ultrafast all-optical switching with magnetic resonances in nonlinear dielectric nanostructures," Nano Lett. 15, 6985-6990 (2015).

11. A. E. Miroshnichenko, A. B. Evlyukhin, Y. F. Yu, R. M. Bakker, A. Chipouline, A. I. Kuznetsov, B. Luk'yanchuk, B. N. Chichkov, and Y. S. Kivshar, "Nonradiating anapole modes in dielectric nanoparticles," Nat. Commun. 6, 8069 (2015).

12. A. A. Basharin, M. Kafesaki, E. N. Economou, C. M. Soukoulis, V. A. Fedotov, V. Savinov, and N. I. Zheludev, "Dielectric metamaterials with toroidal dipolar response," Phys. Rev. X 5, 011036 (2015).

13. B. Luk'yanchuk, R. Paniagua-Domínguez, A. I. Kuznetsov, A. E. Miroshnichenko, and Y. S. Kivshar, "Hybrid anapole modes of high-index dielectric nanoparticles," Phys. Rev. A 95, 063820 (2017).

14. B. Luk'yanchuk, R. Paniagua-Domínguez, A. I. Kuznetsov, A. E. Miroshnichenko, and Y. S. Kivshar, "Suppression of scattering for small dielectric particles: anapole mode and invisibility," Phil. Trans. R. Soc. A 375, 20160069 (2017).

15. S. E. Svyakhovskiy, V. V. Ternovski, and M. I. Tribelsky, "Transient effects at resonant light scattering by particles: Anapole as a storage for" frozen light"?" arXiv preprint arXiv:1708.01436 (2017).

16. J. S. T. Gongora, G. Favraud, and A. Fratalocchi, "Fundamental and high-order anapoles in all-dielectric metamaterials via fano-feshbach modes competition," Nanotechnology 28, 104001 (2017).

17. K. V. Baryshnikova, D. A. Smirnova, B. S. Luk'yanchuk, and Y. S. Kivshar, "Optical anapoles: Concepts and applications," Adv. Opt. Mater. p. 1801350 (2019).

18. Y. B. Zel'Dovich, "Electromagnetic interaction with parity violation," Sov. Phys. JETP 6, 1184-1186 (1958).

19. G. Afanasiev and V. Dubovik, "Some remarkable charge-current configurations," Phys. Part. Nucl. 29, 891-945 (1998).

20. N. Papasimakis, V. Fedotov, V. Savinov, T. Raybould, and N. Zheludev, "Electromagnetic toroidal excitations in matter and free space," Nat. Mater. 15, 263 (2016).

21. G. Grinblat, Y. Li, M. P. Nielsen, R. F. Oulton, and S. A. Maier, "Enhanced third harmonic generation in single germanium nanodisks excited at the anapole mode," Nano Lett. 16, 4635-4640 (2016).

22. G. Grinblat, Y. Li, M. P. Nielsen, R. F. Oulton, and S. A. Maier, "Efficient third harmonic generation and nonlinear subwavelength imaging at a higher-order anapole mode in a single germanium nanodisk," ACS Nano 11, 953-960 (2016).

23. L. Wei, Z. Xi, N. Bhattacharya, and H. P. Urbach, "Excitation of the radiationless anapole mode," Optica 3, 799-802 (2016).

24. R. Wang and L. Dal Negro, "Engineering non-radiative anapole modes for broadband absorption enhancement of light," Opt. Express 24, 19048-19062 (2016).

25. M. Timofeeva, L. Lang, F. Timpu, C. Renaut, A. Bouravleuv, I. V. Shtrom, G. Cirlin, and R. Grange, "Anapoles in free-standing iii-v nanodisks enhancing second-harmonic generation,” Nano Lett. 18, 3695-3702 (2018).

26. D. Rocco, V. F. Gili, L. Ghirardini, L. Carletti, I. Favero, A. Locatelli, G. Marino, D. N. Neshev, M. Celebrano, M. Finazzi, G. Leo, and C. De Angelis, "Tuning the second-harmonic generation in algaas nanodimers via non-radiative state optimization," Photonics Res. 6, B6-B12 (2018).

27. Y. Yang, V. A. Zenin, and S. I. Bozhevolnyi, "Anapole-assisted strong field enhancement in individual all-dielectric nanostructures," ACS Photonics 5, 1960-1966 (2018).

28. D. G. Baranov, R. Verre, P. Karpinski, and M. Käll, "Anapole-enhanced intrinsic raman scattering from silicon nanodisks," ACS Photonics 5, 2730-2736 (2018).

29. L. Xu, M. Rahmani, K. Z. Kamali, A. Lamprianidis, L. Ghirardini, J. Sautter, R. Camacho-Morales, H. Chen, M. Parry, I. Staude, G. Zhang, D. Neshev, and A. E. Miroshnichenko, "Boosting third-harmonic generation by a mirror-enhanced anapole resonator," Light. Sci. \& Appl. 7, 44 (2018).

30. I. Fernandez-Corbaton, S. Nanz, and C. Rockstuhl, "On the dynamic toroidal multipoles from localized electric current distributions," Sci. Rep. 7, 7527 (2017).

31. R. Alaee, C. Rockstuhl, and I. Fernandez-Corbaton, "Exact multipolar decompositions with applications in nanophotonics," Adv. Opt. Mater. 7, 1800783 (2019).

32. D. A. Powell, "Interference between the modes of an all-dielectric meta-atom," Phys. Rev. Appl. 7, 034006 (2017).

33. B. Stout and R. McPhedran, "Egocentric physics: just about mie," EPL 119, 44002 (2017).

34. V. Grigoriev, A. Tahri, S. Varault, B. Rolly, B. Stout, J. Wenger, and N. Bonod, "Optimization of resonant effects in nanostructures via weierstrass factorization," Phys. Rev. A 88, 011803 (2013).

35. R. Colom, R. McPhedran, B. Stout, and N. Bonod, "Modal expansion of the scattered field: Causality, nondivergence, and nonresonant contribution,” Phys. Rev. B 98, 085418 (2018).

36. H. Hulst, Light scattering by small particles, Structure of matter series (Wiley, 1957). 
37. C. F. Bohren and D. R. Huffman, Absorption and scattering of light by small particles, Wiley science paperback series (Wiley, 1983).

38. W. Chew, Waves and fields in inhomogeneous media (Van Nostrand Reinhold, 1990).

39. B. Stout, A. Devilez, B. Rolly, and N. Bonod, "Multipole methods for nanoantennas design: applications to yagi-uda configurations," J. Opt. Soc. Am. B 28, 1213-1223 (2011).

40. K. D. Kokkotas and B. G. Schmidt, “Quasi-normal modes of stars and black holes,” Living Rev. Relativ. 2, 2 (1999).

41. R. K. Chang and A. J. Campillo, Optical processes in microcavities, vol. 3 (World scientific, 1996).

42. C. Sauvan, J.-P. Hugonin, I. Maksymov, and P. Lalanne, "Theory of the spontaneous optical emission of nanosize photonic and plasmon resonators," Phys. Rev. Lett. 110, 237401 (2013).

43. B. Vial, F. Zolla, A. Nicolet, and M. Commandré, "Quasimodal expansion of electromagnetic fields in open two-dimensional structures," Phys. Rev. A 89, 023829 (2014).

44. P. T. Kristensen, R.-C. Ge, and S. Hughes, "Normalization of quasinormal modes in leaky optical cavities and plasmonic resonators," Phys. Rev. A 92, 053810 (2015).

45. X. Zambrana-Puyalto and N. Bonod, "Purcell factor of spherical mie resonators," Phys. Rev. B 91, 195422 (2015).

46. E. Muljarov and W. Langbein, "Exact mode volume and purcell factor of open optical systems," Phys. Rev. B 94, 235438 (2016).

47. E. Lassalle, N. Bonod, T. Durt, and B. Stout, "Interplay between spontaneous decay rates and lamb shifts in open photonic systems," Opt. Lett. 43, 1950-1953 (2018).

48. P. Lalanne, W. Yan, K. Vynck, C. Sauvan, and J.-P. Hugonin, "Light interaction with photonic and plasmonic resonances," Laser \& Photonics Rev. 12, 1700113 (2018).

49. W. Yan, R. Faggiani, and P. Lalanne, "Rigorous modal analysis of plasmonic nanoresonators," Phys. Rev. B 97, 205422 (2018).

50. S. Fan, W. Suh, and J. D. Joannopoulos, "Temporal coupled-mode theory for the fano resonance in optical resonators," J. Opt. Soc. Am. A 20, 569-572 (2003).

51. Z. Ruan and S. Fan, "Temporal coupled-mode theory for fano resonance in light scattering by a single obstacle," The J. Phys. Chem. C 114, 7324-7329 (2009).

52. Z. Ruan and S. Fan, "Temporal coupled-mode theory for light scattering by an arbitrarily shaped object supporting a single resonance," Phys. Rev. A 85, 043828 (2012).

53. U. Fano, "Effects of configuration interaction on intensities and phase shifts," Phys. Rev. 124, 1866 (1961).

54. B. Luk'yanchuk, N. I. Zheludev, S. A. Maier, N. J. Halas, P. Nordlander, H. Giessen, and C. T. Chong, “The fano resonance in plasmonic nanostructures and metamaterials," Nat. Mater. 9, 707-715 (2010).

55. A. E. Miroshnichenko and Y. S. Kivshar, "Fano resonances in all-dielectric oligomers," Nano Lett. 12, 6459-6463 (2012).

56. M. V. Rybin, K. B. Samusev, I. S. Sinev, G. Semouchkin, E. Semouchkina, Y. S. Kivshar, and M. F. Limonov, "Mie scattering as a cascade of fano resonances," Opt. Express 21, 30107-30113 (2013).

57. V. Grigoriev, S. Varault, G. Boudarham, B. Stout, J. Wenger, and N. Bonod, "Singular analysis of fano resonances in plasmonic nanostructures," Phys. Rev. A 88, 063805 (2013).

58. M. V. Rybin, D. S. Filonov, P. A. Belov, Y. S. Kivshar, and M. F. Limonov, "Switching from visibility to invisibility via fano resonances: theory and experiment," Sci. Rep. 5, 8774 (2015).

59. A.-L. Fehrembach, B. Gralak, and A. Sentenac, "Vectorial model for guided-mode resonance gratings," Phys. Rev. A 97, 043852 (2018).

60. B. Stout, R. Colom, N. Bonod, and R. McPhedran, "Eigenstate normalization for open and dispersive systems," arXiv preprint arXiv:1903.07183 (2019). 